\title{
INFARCT SIZE AND LOCATION DETERMINE DEVELOPMENT OF MITRAL REGURGITATION IN THE SHEEP MODEL
}

Joseph H. Gorman III, MD

Robert C. Gorman, MD

Theodore Plappert, CVT

Benjamin M. Jackson, MS

Yugi Hiramatsu, MD

Martin G. St. John-Sutton, FRCP

L. Henry Edmunds, Jr., MD
Objective: This study tests the hypothesis that neither small nor large myocardial infarctions that include the anterior papillary muscle produce mitral regurgitation in sheep. Methods: Coronary arterial anatomy to the anterior left ventricle and papillary muscle was determined by dye injection in 41 sheep hearts and by triphenyl tetrazolium chloride in 13. Development of acute or chronic mitral regurgitation and changes in left ventricular dimensions were studied by use of transdiaphragmatic echocardiography in 21 sheep after infarction of $24 \%$ and $33 \%$ of the anterior left ventricular mass. These data were compared with previous data from large and small posterior left ventricular infarctions. Results: Ligation of two diagonal arteries infarcts $24 \%$ of the left ventricular mass and $82 \%$ of the anterior papillary muscle. Ligation of both diagonals and the first circumflex branch infarcts $33 \%$ of the left ventricle and all of the anterior papillary muscle. Neither infarction causes mitral regurgitation, although left ventricular cavity dimensions increase significantly at end systole. After the smaller infarction, the left ventricular cavity enlarges $150 \%$ over 8 weeks without mitral regurgitation. Conclusions: In sheep small and large infarctions of the anterior wall that include the anterior papillary muscle do not produce either acute or chronic mitral regurgitation despite left ventricular dilatation. In contrast large posterior infarctions produce immediate mitral regurgitation owing to asymmetric annular dilatation and discoordination of papillary muscle relationships to the valve. After small posterior infarctions that include the posterior papillary muscle, mitral regurgitation develops because of annular and ventricular dilatation during remodeling. (J Thorac Cardiovasc Surg 1998;115:615-22) schemic mitral regurgitation (IMR) is a prevalent disease ${ }^{1}$ that is caused by acute or chronic myocardial infarction. ${ }^{2}$ The disease process does not directly involve the mitral valve leaflets or chordae, but it does involve other components of the mitral apparatus, especially the ventricle, papillary muscles, and anulus. ${ }^{3}$ IMR is differentiated from mitral regurgitation (MR) associated with coronary artery

From the Department of Surgery, School of Medicine, University of Pennsylvania, Philadelphia, Pa.

Supported by grant HL 36308 from the National Heart, Lung, and Blood Institute, National Institutes of Health, Bethesda, Md.

Received for publication July 22, 1997; revisions requested Sept. 11, 1997; revisions received Sept. 19, 1997; accepted for publication Sept. 30, 1997.

Address for reprints: L. Henry Edmunds Jr., MD, Department of Surgery, Six Silverstein, Hospital of the University of Pennsylvania, 3400 Spruce St., Philadelphia, PA 19104.

Copyright (C) 1998 by Mosby, Inc.

$0022-5223 / 98 \$ 5.00+0 \quad \mathbf{1 2 / 1 / 8 6 7 2 3}$ disease but caused by other pathologic conditions. ${ }^{3}$ Both acute and chronic IMR are encountered clinically $^{1,3,4}$; severe MR associated with acute infarction is life-threatening and usually requires emergency operation for survival. ${ }^{3-5}$ Chronic MR is a disease of ventricular remodeling. ${ }^{3}$ Posterior myocardial infarction, which produces MR more often than anterior infarction, 2,6 often includes the posterior papillary muscle and is associated with mild to moderate ventricular dilatation. In contrast anterior infarction that produces chronic IMR often does not involve the anterior papillary muscle ${ }^{6}$ and is associated with more massive ventricular dilatation. ${ }^{6} \mathrm{Be}-$ cause the geometric deformations that cause the valve to leak are not well understood, repair of severe IMR is less satisfactory than valve replacement $^{7}$; and, more often than not, mild to moderate IMR is not addressed at all during myocardial revascularization procedures. 1,3

The development of a sheep model of acute and chronic $\mathrm{IMR}^{8}$ and new imaging techniques of three- 
dimensional echocardiography, ${ }^{9}$ marker angiography, ${ }^{10}$ and sonomicrometry array localization ${ }^{11}$ provide technology to determine the specific geometric deformations that cause IMR. An understanding of these deformations is prerequisite to successful correction of IMR. This study further develops the sheep model of IMR by correlating the development of IMR to small and large anterior and posterior infarctions.

\section{Methods}

Anatomic studies. Coronary arterial anatomy was determined in 41 excised sheep hearts that were obtained from a local slaughterhouse and flushed with heparinized saline solution $(10 \mathrm{U} / \mathrm{ml})$ and stored in refrigerated saline solution containing $1 \mathrm{gm}$ chloramphenicol. All these hearts and those used in the in vivo studies (below) were type $\mathrm{A}$ in that the homonymous artery supplied the left ventricular apex. ${ }^{8}$ Because sheep lack preformed collateral vessels, ${ }^{12,13} 10 \mathrm{ml}$ of methylene blue dye was injected by hand into specific vessels to determine the myocardial territory supplied by each vessel or vessel group. After injection, stained hearts were sliced transaxially at $5 \mathrm{~mm}$ intervals. Each slice was traced onto acetate paper and carefully marked to differentiate stained and unstained myocardium. Tracings were digitized (Truegrid, Houston Instruments, Houston, Tex.) and the percentage of myocardium served by each branch was calculated with customized software (Asyst Software Technologies, Rochester, N.Y.).

Six injections of methylene blue dye were made into the first diagonal artery (D1) and five injections were made into the second diagonal (D2). The first obtuse marginal of the circumflex artery (OM1) was injected in five hearts. In six hearts the combination of D1 and OM1 were simultaneously injected. Seven injections were made simultaneously into the first (D1) and second (D2) diagonal branches of the homonymous artery (equivalent to the human left anterior descending coronary artery), and seven injections were made into D1, D2, and OM1.

The percentage of anterior papillary muscle mass supplied by the two diagonal arteries was determined in five additional slaughterhouse sheep hearts by volume displacement. Methylene blue dye $(10 \mathrm{ml})$ was injected into D1 and D2. The anterior papillary muscle was carefully excised and included the contiguous left ventricular wall. Stained muscle was sharply demarcated and was easily separated from unstained muscle; the volume of each portion was determined by saline displacement in a graduated cylinder. The percentage of the anterior muscle mass supplied by D1, and D2 was calculated from the volume measurements.

In vivo studies. In compliance with the Guide for the Care and Use of Laboratory Animals ${ }^{+}$published by the National Institutes of Health (NIH publication 85-23, revised 1985) 21 Dorsett hybrid sheep between 35 and 45 $\mathrm{kg}$ and bred for laboratory use were induced with sodium thiopental (12.5 mg/kg intravenously) and intubated. Anesthesia was maintained with $1 \%$ to $2 \%$ isoflurane in oxygen. All animals received glycopyrrolate (INN: glycopyrronium bromide) ( $0.4 \mathrm{mg}$ intravenously) 1 hour before incision; surviving animals also received cefazolin (1 gm intravenously) before and 1 day after operation. All animals had left thoracotomy with sterile or clean ${ }^{8}$ technique. Animals received magnesium (1 gm intravenously) and lidocaine (3 $\mathrm{mg} / \mathrm{kg}$ intravenously) before ligating selected coronary arteries and had an infusion of lidocaine ( $2 \mathrm{mg} / \mathrm{min}$ ) for 60 minutes afterward. The wound was closed with a single chest tube in surviving animals; when the sheep awakened, the endotracheal tube and chest tube were removed. These animals received flunixin meglumine (1 mg/kg intramuscularly) postoperatively for pain.

Quantitative, subdiaphragmatic echocardiographic studies were obtained before and after infarction in all sheep and were repeated at 4 and 8 weeks after infarction in 8 of the 21 animals. The reproducibility of twodimensional echocardiographic measurements in sheep over time has been previously reported. ${ }^{14}$ Briefly left ventricular (LV) short axis images acquired subdiaphragmatically are selected at baseline by use of internal landmarks (i.e., papillary muscles, tips of mitral valve leaflets, circular shape of the cavity, and thickness of ventricular walls). This combination of objective criteria enabled acquisition of true short axis slices with a high degree of certainty. After infarction, short axis images were not completely circular and wall thickness was not uniform; thus images were acquired only when papillary muscles and tips of mitral leaflets were aligned in plane. ${ }^{+}$The degree of mitral regurgitation was determined by color flow Doppler velocity mapping by use of previously described criteria. ${ }^{15}$

In 13 sheep D1 and D2 were ligated; five of these animals were killed after echocardiographic data were obtained. In 8 sheep D1, D2, and OM1 were ligated; all of these sheep were killed after the acute study. The percentage of infarcted myocardium in the 13 sheep killed shortly after infarction was determined after infusion of triphenyl tetrazolium chloride for 30 minutes as described previously. ${ }^{16}$ In the 8 sheep that were followed for 8 weeks after ligation of D1 and D2 echocardiograms and color flow Doppler velocity maps were obtained during a sterile laparotomy by use of right or left subcostal or midline upper abdominal incisions. ${ }^{17} \mathrm{LV}$ dimensions and infarct size were determined from short axis echocardiograms at end systole and end diastole at each time interval. Infarct size was assessed echocardiographically as the length of endocardium that was akinetic or dyskinetic. At autopsy the percentage of anterior papillary muscle infarction was determined by volume displacement.

Statistics. Mean and standard deviations were calculated. For acute studies measurements before and after infarction were compared by the paired $t$ statistic and $p$ values were calculated using SPSS 6.0 software (SPSS, Inc., Chicago, Ill.). For chronic studies, measurements after infarction were compared with before infarction using analysis of variance for repeated measurements with SPSS software. $P$ values for differences before and after infarction were calculated by the paired $t$ statistic with the Bonferroni correction.

\section{Results}

Anatomic studies. Together D1 and D2 supply $24.6 \% \pm 4.7 \%$ of the LV mass in 7 slaughterhouse hearts and $23.9 \% \pm 2.2 \%$ in 5 sheep studied after in 
vivo ligation of both vessels (Table I). The size of the two vessels varies reciprocally between each other, but the territory supplied by D1 and D2 is quite constant (Fig. 1). The percentage of the anterior papillary muscle supplied by D1 and D2 averaged $82.7 \% \pm$ $8.9 \%(n=5)$. The percentage of LV mass supplied by D1, D2, and OM1 averaged $30.5 \% \pm 3.2 \%(n=7)$ in slaughterhouse hearts and $35.2 \% \pm 4.9 \%$ in 8 animals that were killed after ligation of these three vessels. Ligation of D1, D2, and OM1 infarcted all of the anterior papillary muscle in every sheep (Fig. 2).

In vivo studies. No MR developed in 11 of 13 sheep 30 to 60 minutes after the D1, D2 infarction. In one sheep 3+ MR developed immediately; in another 2+ MR that persisted over 8 weeks of follow-up occurred. Acute changes in ventricular dimensions of these two sheep did not differ from the others, and no explanation for development of MR was found. No MR developed in the 8 sheep after ligature of D1, D2, and OM1 coronary arterial branches.

Changes in LV dimensions after infarction in the three groups of animals are presented in Table II and Figs. 2 through 4. Diastolic short axis dimensions did not significantly increase immediately after either small (D1, D2) or large (D1, D2, OM1) anterior infarctions. In contrast end-systolic cavity diameter and area significantly increased by $13.8 \%$ and $33.3 \%$ immediately after ligation of D1, D2 and $12.5 \%$ and $34.2 \%$ after ligation of D1, D2, and OM1 (Table II).

Greater increases in ventricular dimensions occurred during the 8 weeks after occlusion of D1, D2 (Table II, Fig. 4). By 4 weeks the entire left ventricle was dilated both at end systole and at end diastole and continued to enlarge further during the next 4 weeks. MR did not develop despite an increase in end-systole cavity area of 150\% (Table II).

\section{Discussion}

The roles of papillary muscle infarction and annular dilatation in the pathogenesis of IMR are explained by comparing sizes and locations of ovine infarctions (Table III). In previous studies large acute posterior infarctions of $32 \%$ to $35 \%$ of the LV mass include the posterior papillary muscle, produce severe MR and asymmetric dilatation of the posterior anulus that causes a small $(9.3 \%)$ but significant increase in annular area. ${ }^{17,18}$ Large acute anterior infarctions of $31 \%$ to $35 \%$ of the LV include the anterior papillary muscle, do not produce MR, and probably do not increase annular area for the following reasons. In dogs systolic constriction of the normal anulus is minimal along the anterior anu-
Table I. Relationship between ovine coronary anatomy and amount of left ventricular mass supplied by single vessels and combinations of arteries in sheep

\begin{tabular}{llcc}
\hline & Coronary arteries & $N$ & $\begin{array}{c}\text { Infarct size } \\
(\%)\end{array}$ \\
\hline Slaughterhouse hearts & D1 & 6 & $16.5 \pm 2.9$ \\
& D2 & 5 & $16.0 \pm 3.2$ \\
& OM1 & 5 & $12.3 \pm 2.0$ \\
& D1,OM1 & 6 & $19.2 \pm 4.7$ \\
In vivo hearts & D1,D2 & 7 & $24.6 \pm 4.7$ \\
& D1,D2,OM1 & 6 & $31.0 \pm 3.0$ \\
& D1,D2 & 5 & $23.9 \pm 2.2$ \\
& D1,D2,OM1 & 8 & $35.2 \pm 4.9$ \\
\hline
\end{tabular}

lus. ${ }^{19}$ The anterior anulus is reinforced by the fibrous trigone. Anterior infarctions are located closer to the apex than the base. An I-beam* effect of the anterior septum abutting the right and left ventricular walls probably limits displacement of the anterior papillary muscle.

Large posterior acute infarctions produce immediate MR because of the combination of asymmetric annular dilatation and papillary muscle infarction that together distort the spatial relationships of leaflet coaptation. ${ }^{14,18}$ Large anterior acute infarctions do not enlarge or distort the mitral anulus sufficiently to produce acute IMR even though the anterior papillary muscle is infarcted.

Small anterior ovine infarctions involve $24 \%$ of the LV mass and greater than $80 \%$ of the anterior papillary muscle, do not cause acute or chronic IMR, but produce massive LV dilatation over time. Small posterior ovine infarctions involve $21 \%$ to $24 \%$ of the LV mass, may or may not involve the posterior papillary muscle, do not produce acute MR, but may produce MR over time if the posterior papillary muscle is involved. ${ }^{8}$ Acute coronary occlusion produces an immediate loss of myocyte shortening ${ }^{20}$; thus the posterior papillary muscle stops contracting immediately after ligation of its blood supply, but this loss does not produce MR. ${ }^{8}$ As the anulus and LV dilate over time, postinfarction ventricular remodeling produces MR and a large increase in annular area and LV volume.* Small posterior infarctions that do not include the posterior papillary muscle produce ventricular dilatation but min-

\footnotetext{
*Gorman JH III, Gorman RC, Kelley ST, Gikakis N, Plappert T, St. John-Sutton MG, et al. Pathologic geometry of chronic mitral regurgitation after posterior myocardial infarction. Manuscript in preparation.
} 

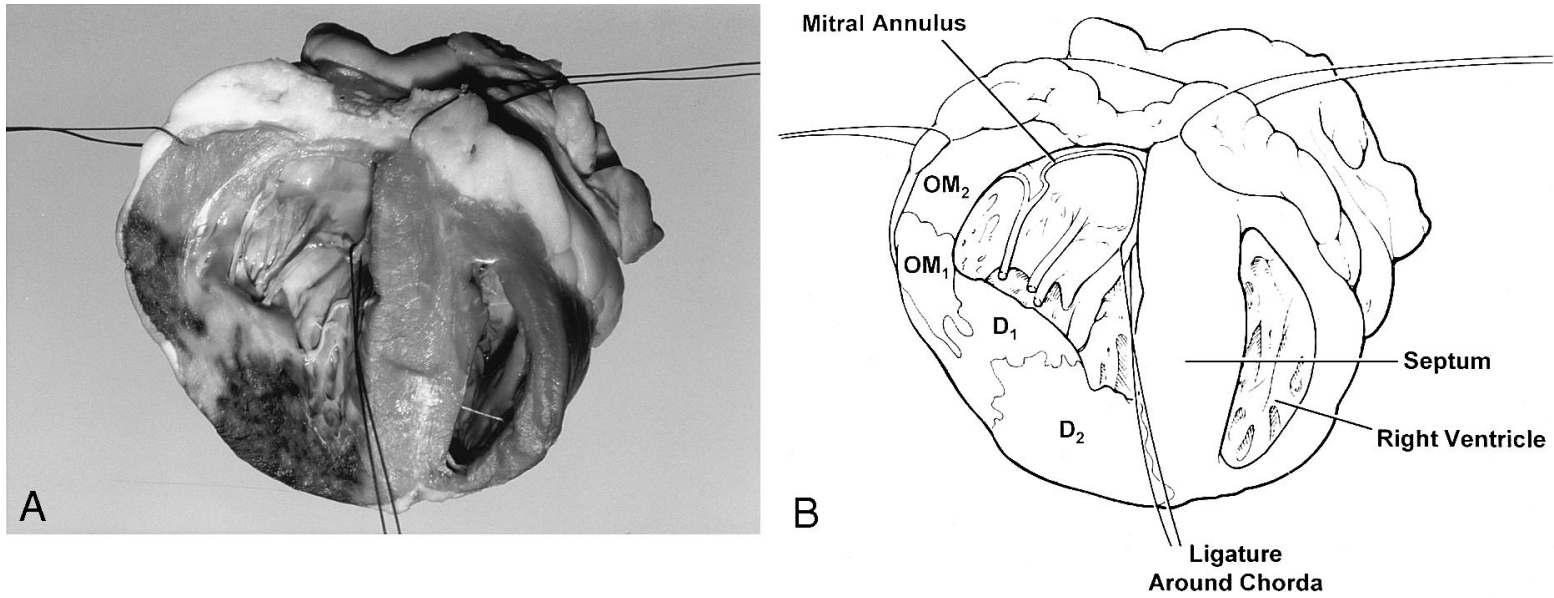

Fig. 1. A, Photograph of the ventricular surface of the anterolateral wall after separate methylene blue injections of D2 and OM1 in a slaughterhouse heart. The injected heart was cut approximately along a right anterior oblique axis to demonstrate the location and sharp outlines produced by the dye injection of the myocardium supplied by both diagonals and the first two circumflex marginal coronary arteries. The anterior leaflet of the mitral valve partially remains, but the tip of the anterior papillary muscle $(A P M)$ has been removed. B, Drawing of the photograph in $A$ with labels. OM2 and OM1, Myocardium supplied by circumflex marginals OM2 (unstained) and OM1 (stained). D1 and D2, Myocardium supplied by diagonal coronary arteries D1 (unstained) and D2 (stained).


Fig. 2. Short axis echocardiograms before (A, B) and 30 to 60 minutes after $(\mathbf{C}, \mathbf{D})$ ligation of D1, D2, and OM1 at end diastole $(\mathbf{A}, \mathbf{C})$ and end systole $(\mathbf{B}, \mathbf{D})$. The cross-sectional cavity area at end diastole is not increased by adding OM1 to ligation of D1, D2 (see Fig. 3, Table II). Arrow in D points to the approximate center of the infarction. 

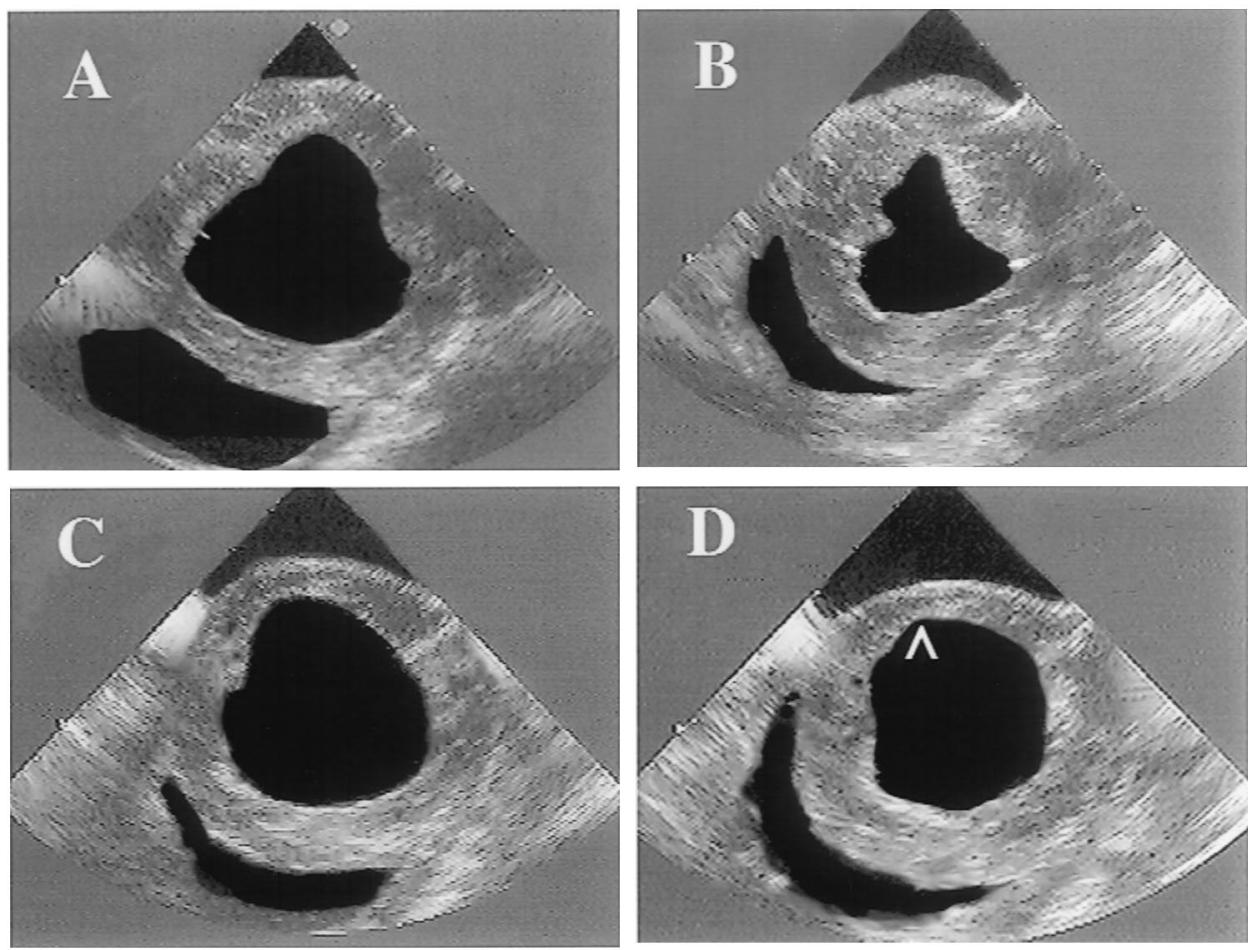

Fig. 3. Short axis echocardiograms before (A, B) and 30 to 60 minutes after (C, D) ligation of D1, D2 at end diastole $(\mathbf{A}, \mathbf{C})$ and end systole $(\mathbf{B}, \mathbf{D})$. Note that most of the increase in cross-sectional cavity area occurs at end systole. Arrow in panel $D$ indicates the approximate center of the infarction.

Table II. Changes in total area, cavity area, and short axis cavity diameter at end diastole and end systole and total end-diastolic endocardial perimeter and end-systolic length (longitudinal axis) of the akinetic segment

\begin{tabular}{|c|c|c|c|c|c|c|c|c|c|}
\hline \multirow[b]{2}{*}{ Arteries } & \multirow[b]{2}{*}{ Infarction } & \multicolumn{3}{|c|}{ End diastole } & \multicolumn{3}{|c|}{ End systole } & \multirow[b]{2}{*}{$\begin{array}{c}\text { Endocardial } \\
\text { perimeter } \\
(\mathrm{cm})\end{array}$} & \multirow[b]{2}{*}{$\begin{array}{l}\text { Akinetic } \\
\text { length } \\
(\mathrm{cm})\end{array}$} \\
\hline & & $\begin{array}{c}\text { Total area } \\
\left(\mathrm{cm}^{2}\right)\end{array}$ & $\begin{array}{l}\text { Cavity area } \\
\qquad\left(\mathrm{cm}^{2}\right)\end{array}$ & $\begin{array}{l}\text { Cavity } \\
\text { diameter } \\
(\mathrm{cm})\end{array}$ & $\begin{array}{l}\text { Total area } \\
\left(\mathrm{cm}^{2}\right)\end{array}$ & $\begin{array}{c}\text { Cavity area } \\
\left(\mathrm{cm}^{2}\right)\end{array}$ & $\begin{array}{l}\text { Cavity } \\
\text { diameter } \\
(\mathrm{cm})\end{array}$ & & \\
\hline \multirow[t]{2}{*}{ D1,D2,OM1 acute } & Before & $27.3 \pm 5.0$ & $14.3 \pm 2.9$ & $4.2 \pm 0.5$ & $22.5 \pm 3.9$ & $8.2 \pm 1.5$ & $3.2 \pm 0.3$ & $13.7 \pm 1.3$ & 0 \\
\hline & After & $29.0 \pm 6.0$ & $16.5 \pm 4.0$ & $4.3 \pm 0.3$ & $24.8 \pm 4.7$ & $11.0=$ & $3.6 \pm 0.3 \dagger$ & $14.6 \pm 1.7$ & $4.6 \pm 1.1$ \\
\hline \multirow[t]{2}{*}{ D1,D2 acute } & Before & $25.3 \pm 2.9$ & $13.4 \pm 3.0$ & $4.0 \pm 0.7$ & $20.2 \pm 3.0$ & $6.9 \pm 1.7 \dagger$ & $2.9 \pm 0.2 \dagger$ & $13.2 \pm 1.5$ & 0 \\
\hline & After & $27.4 \pm 2.6$ & $15.0 \pm 2.4$ & $4.4 \pm 0.5$ & $22.5 \pm 1.9$ & $9.2 \pm 1.2$ & $3.3 \pm 0.4$ & $13.9 \pm 1.0$ & $3.3 \pm 0.7$ \\
\hline \multirow[t]{4}{*}{ D1,D2 chronic } & Before & $22.2 \pm 2.6$ & $11.3 \pm 1.7$ & $3.5 \pm 0.4$ & $17.6 \pm 3.1$ & $5.9 \pm 1.7$ & $2.5 \pm 0.5$ & $12.0 \pm 1.0$ & 0 \\
\hline & After & $25.7 \pm 2.7$ & $13.5 \pm 1.9$ & $4.1 \pm 0.4 \dagger$ & $21.5 \pm 2.0$ & $7.2 \pm 0.9$ & $2.9 \pm 0.2$ & $13.5 \pm 1.0$ & $2.3 \pm 1.6$ \\
\hline & Four weeks & $30.6 \pm 3.0 \ddagger$ & $17.7 \pm 4.0^{*}$ & $4.6 \pm 0.5 \ddagger$ & $26.9 \pm 4.2 \ddagger$ & $13.6 \pm 3.5 \ddagger$ & $4.0 \pm 0.4 \ddagger$ & $15.2 \pm 1.7 \ddagger$ & $4.8 \pm 0.7 \ddagger$ \\
\hline & Eight weeks & $34.2 \pm 6.6 \ddagger$ & $19.6 \pm 6.2 \ddagger$ & $4.8 \pm 0.6 \ddagger$ & $30.1 \pm 6.2 \ddagger$ & $14.8 \pm 4.6 \ddagger$ & $4.2 \pm 0.6 \ddagger$ & $15.9 \pm 2.3 \ddagger$ & $4.9 \pm 0.7 \ddagger$ \\
\hline
\end{tabular}

${ }^{*} p<0.01$ for indicated time point versus before infarction by paired $t$ test (Bonferroni correction).

$\dagger p<0.05$ for indicated time point versus before infarction by paired $t$ test (Bonferroni correction).

$\ddagger p<0.001$ for indicated time point versus before infarction by paired $t$ test (Bonferroni correction).

imal annular dilatation and no MR.* Thus when the infarct is small (21\% to $24 \%$ ), ischemic MR in sheep requires annular dilatation, papillary muscle infarction, and posterior location. Neither small nor large anterior infarctions produce IMR in sheep even though the LV dilates and all or most of the anterior papillary muscle is necrotic.

In patients variability of coronary arterial anatomy and the heterogeneity of myocardial infarctions preclude correlation between the size and location 

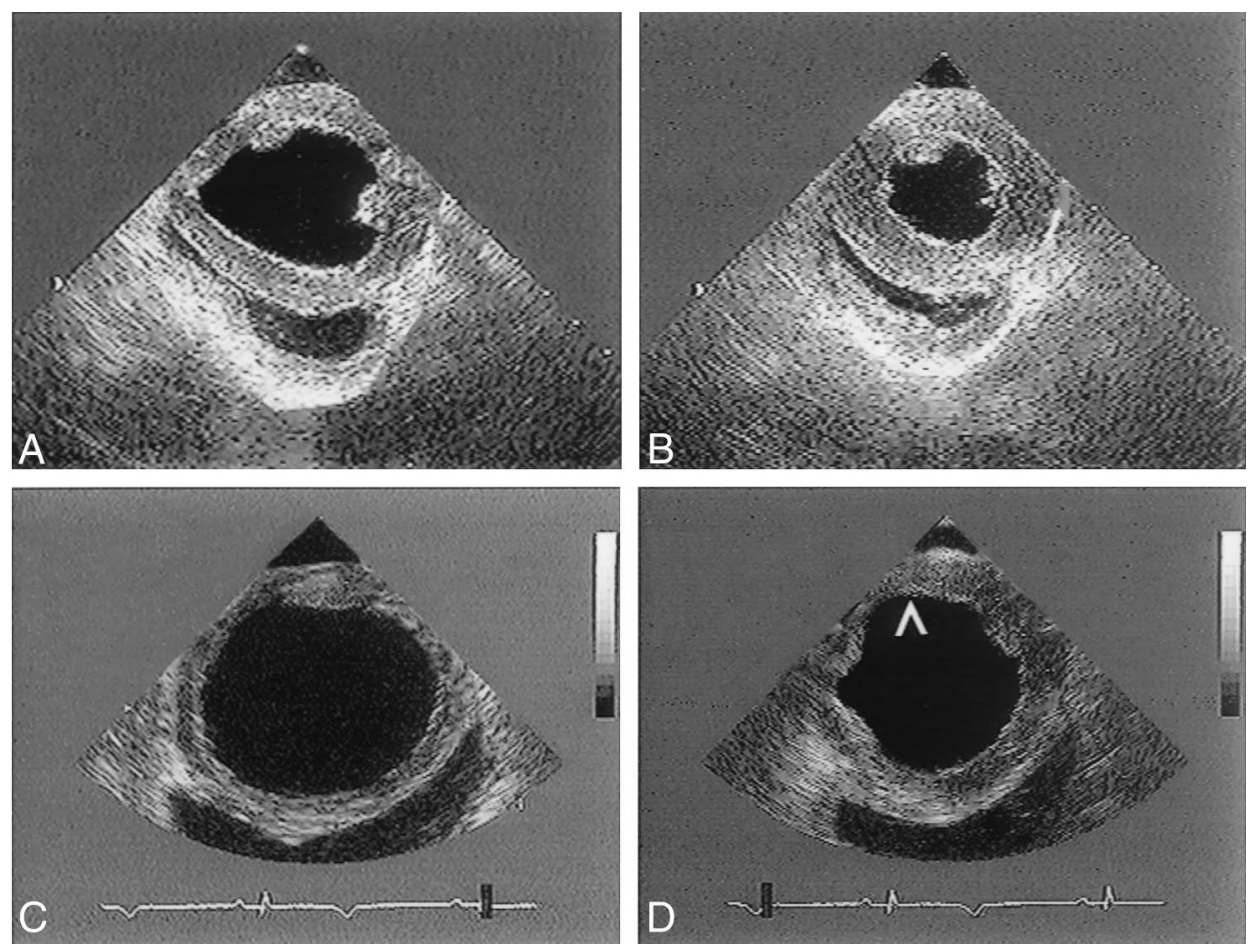

Fig. 4. Short axis echocardiograms before $(\mathbf{A}, \mathbf{B})$ and 8 weeks after $(\mathbf{C}, \mathbf{D})$ ligation of D1, D2, and OM1 at end diastole (A, C) and end systole (B, D). Although end-diastolic cavity area has increased $73 \% 8$ weeks after infarction, end-systolic cavity area has increased $151 \%$ (Table II). Arrow indicates the approximate center of the infarction.

Table III. Summary of variables related to ischemic mitral regurgitation in sheep. Citations for posterior myocardial infarction given in the first column. Qualitative estimates of the magnitude of mitral regurgitation and left ventricular dilation are given in a scale of 1 to $4+$

\begin{tabular}{|c|c|c|c|c|c|c|}
\hline Location of infarct & $\begin{array}{l}\text { Time after } \\
\text { infarct }\end{array}$ & $\begin{array}{c}\text { Size of } \\
\text { infarct } \\
(\%)\end{array}$ & $M R$ & $\begin{array}{l}\text { Papillary } \\
\text { muscle } \\
\text { necrosis }\end{array}$ & $\begin{array}{l}\text { Annular } \\
\text { dilatation }\end{array}$ & $\begin{array}{c}\text { Left } \\
\text { ventricular } \\
\text { dilatation }\end{array}$ \\
\hline \multicolumn{7}{|l|}{ Posterior infarctions } \\
\hline OM1,OM2 (8) & Acute & 24 & No & No & No & No \\
\hline (8) & Late & & No & No & Unknown & $4+$ \\
\hline $\mathrm{OM} 2, \mathrm{OM} 3$ & Acute & 21 & No & Yes & No & No \\
\hline Complete $(8,21)$ & & & & & & \\
\hline$(21)^{1}$ & Late & & $3+-4+$ & Yes & $4+$ & $4+$ \\
\hline $\mathrm{OM} 2, \mathrm{OM} 3$ & Acute & 21 & No & No & No & No \\
\hline $\begin{array}{l}\text { Incomplete (21) } \\
(21)\end{array}$ & Late & & No & No & No & $2+$ \\
\hline $\begin{array}{l}\text { OM2,OM3,PDA } \\
(17,19)\end{array}$ & Acute & $32-35 \%$ & $2+-3+$ & Yes & Asymmetric & $2+$ \\
\hline \multicolumn{7}{|l|}{ Anterior infarctions } \\
\hline $\mathrm{D} 1, \mathrm{D} 2$ & Acute & 24 & No & Yes & Unlikely & $2+$ \\
\hline & Late & & No & Yes & Unlikely & $4+$ \\
\hline D1,D2,OM1 & Acute & 35 & No & Yes & Unlikely & $1+$ \\
\hline
\end{tabular}

$M R$, Mitral regurgitation.

of infarctions and the presence or absence of IMR. ${ }^{21}$ Echocardiograms often show incomplete mitral valve closure in patients with IMR, ${ }^{22}$ and the ventricle is more spherical in shape. ${ }^{23}$ Chronic IMR caused by posterior infarctions is associated with complete infarction of the papillary muscle ${ }^{6}$ and less overall LV dilatation than chronic IMR involving the anterior papillary muscle. ${ }^{22}$ In approximately 
half of the patients with chronic IMR caused by anterior infarctions, the anterior papillary muscle is not involved, ${ }^{2,6}$ but in all patients the left ventricle is markedly dilated. ${ }^{21}$ Because the diameter of the mitral anulus is closely related to LV end-diastolic volume, ${ }^{24,25}$ IMR after anterior infarctions correlates with large ventricular volumes in patients. ${ }^{21}$

Except for papillary muscle rupture, most acute infarctions that produce life-threatening IMR in patients are posterior and involve the posterior papillary muscle., ${ }^{2,3}$ Often the degree of LV dilatation is relatively small unless the ventricle was dilated before the acute infarction. ${ }^{2}$ The anulus is not dilated, ${ }^{2,5}$ and most of these infarctions are associated with single- or double-vessel coronary arterial disease. ${ }^{3}$ When the anterior papillary muscle is infarcted in acute postinfarction MR, usually both the ventricle and anulus are dilated. ${ }^{2}$

The anatomy of sheep and human mitral valves differ in details, but the design is the same and the mechanism of ischemic MR after infarctions of various sizes and locations is probably similar. In sheep most of both papillary muscles is contiguous with the ventricular wall, ${ }^{11}$ chordae are proportionately shorter ${ }^{11}$ and perhaps the importance of papillary muscle shortening is less than in humans. Nevertheless the consistency of ovine coronary arterial anatomy, lack of preformed collateral vessels, and predictability of the size and location of infarctions strips away extraneous variables caused by the heterogeneity of human disease and permits a clearer understanding of the anatomic deformations that lead to acute and chronic IMR. These findings correlate well with patient observations, validate the usefulness of the sheep model, and provide a basis for more effective surgical repair of acute and chronic IMR.

\section{REFERENCES}

1. Hickey M StJ, Smith LR, Muhlbaier LH, Harrell FE Jr, Reves JG, Hinohara T, et al. Current prognosis of ischemic mitral regurgitation. Circulation 1988;78(Suppl):I51-9.

2. Heikkila J. Mitral incompetence as a complication of acute myocardial infarction. Acta Medica Scand Suppl 1967:475:1141.

3. Edmunds LH Jr. Ischemic mitral regurgitation. In: Edmunds LH Jr, editor. Cardiac surgery in the adult. New York: McGraw-Hill; 1997. p. 657-76.

4. Vetter HO, Hetzer R, Schmutzler H, editors. Ischemic mitral incompetence. New York: Springer-Verlag; 1991.

5. Wei JY, Hutchins GM, Bulkley BH. Papillary muscle rupture and fatal acute myocardial infarction. Ann Intern Med 1979;90:149-53.

6. Becker AE. Anatomy of the coronary arteries with respect to chronic ischemic mitral regurgitation. In: Vetter $\mathrm{HO}$, Hetzer $\mathrm{R}$, Schmutzler $\mathrm{H}$, editors. Ischemic mitral incompetence. New York: Springer-Verlag; 1991. p. 17-24.

7. Cohn LH, Rizzo RJ, Adams DH, Couper GS, Sullivan TE, Collins JJ Jr, et al. The effect of pathophysiology on the surgical treatment of ischemic mitral regurgitation: operative and late risks of repair versus replacement. Eur J Cardiothorac Surg 1995;9:568-74.

8. Llaneras MR, Nance ML, Streicher JT, et al. A large animal model of ischemic mitral regurgitation. Ann Thorac Surg 1994;57:432-9.

9. Jiang L, Vazquez de Prada JA, Handschumacher MD, Vuille C, Guerrero JL, Picard MH, et al. Quantitative threedimensional reconstruction of aneurysmal left ventricles: in vitro and in vivo validation with initial clinical application. Circulation 1995;91:222-30.

10. Daughters GT, Sanders WJ, Miller DC, Schwarzkopf A, Mead CW, Ingels NB Jr. A comparison of two analytical systems for 3-D reconstruction from biplane videoradiograms. Proc Comp Cardiol (IEEE) 1989;15:79-82.

11. Gorman JH, Gupta KB, Streicher JT, Gorman RC, Jackson $\mathrm{BM}$, Ratcliffe MB, et al. Dynamic three-dimensional imaging of the mitral valve and left ventricle using rapid sonomicrometry array localization. J Thorac Cardiovasc Surg 1996;112: $712-26$.

12. Vastesaeger MM, Van Der Straeten PP, Friart J, Candaele G, Ghys A, Vemard RM. Les anastomoses intercoronariennes telles qu'elles apparaissent a la coronarographie post mortem. Acta Cardiologica 1957;12:365-401.

13. Schaper W. Collateral circulation of the heart. Amsterdam: North Holland; 1971.

14. Gorman JH III, Gorman RC, Jackson BM, Hiramatsu Y, Gikakis N, Kelley ST, et al. Distortions of the mitral valve in acute ischemic mitral regurgitation. Ann Thorac Surg 1997; 64:1026-31.

15. Abassi AS, Allen MW, DeCristofaro D, Ungar I. Detection and estimation of the degree of mitral regurgitation by range-gated pulsed Doppler echocardiography. Circulation 1980;61:143-7.

16. Markowitz LJ, Savage EB, Ratcliffe MB, Bavaria JE, Kreiner G, Iozzo RV, et al. Large animal model of left ventricular aneurysm. Ann Thorac Surg 1989;48:838-45.

17. Gorman JH III, Jackson BM, Gorman RC, Kelley ST, Gikakis N, Edmunds LH Jr. Papillary muscle discoordination rather than increased annular area facilitates mitral regurgitation after acute posterior myocardial infarction. Circulation 1997;96(Suppl):II124-7.

18. Gorman RC, McCaughan JS, Ratcliffe MB, Gupta KB, Streicher JT, Ferrari VA, et al. Pathogenesis of acute ischemic mitral regurgitation in three dimensions. J Thorac Cardiovasc Surg 1995;109:684-93.

19. Glasson JR, Komeda M, Daughters GT, Niczyporuk MA, Bolger AF, Ingels NB, et al. Three-dimensional regional dynamics of the normal mitral anulus during left ventricular ejection. J Thorac Cardiovasc Surg 1996;111:574-85.

20. Heyndrickx GR, Baig H, Nellens P, Leusen I, Fishbein MC, Vatner SF. Depression of regional blood flow and wall thickening after brief coronary occlusions. Am J Physiol 1978;234:H653-9.

21. Frantz E, Weininger F, Oswald H, Fleck E. Predictors for mitral regurgitation in coronary artery disease. In: Vetter 
HO, Hetzer R, Schmutzler H, editors. Ischemic mitral incompetence. New York: Springer-Verlag; 1991. p. 57-73.

22. Godley RW, Wann IS, Rogers EW, Feigenbaum H, Weyman AE. Incomplete mitral leaflet closure in patients with papillary muscle dysfunction. Circulation 1981;63:565-71.

23. Kono T, Sabbah HN, Stein PD, Brymer JF, Khaja F. Left ventricular shape as a determinant of functional mitral regurgitation in patients with severe heart failure secondary to either coronary artery disease or idiopathic dilated cardiomyopathy. Am J Cardiol 1991;68:355-9.
24. Breisblatt WM, Cergueira M, Francis CK, Plankey M, Zaret $\mathrm{BL}$, Berger HJ. Left ventricular function in ischemic mitral regurgitation: a precatheterization assessment. Am Heart J 1987;115:77-82.

25. Erbel R, Drexler M, Mohr-Kahaly S, Wittlich N, Meyer J. Diagnostic value of transesophageal echocardiography in patients with coronary artery disease and mitral insufficiency. In: Vetter HO, Hetzer R, Schmutzler H, editors. Ischemic mitral incompetence. New York: Springer-Verlag; 1991. p. 89-98.

\section{Bound volumes available to subscribers}

Bound volumes of The Journal of Thoracic and Cardiovascular Surgery are available to subscribers (only) for the 1998 issues from the Publisher, at a cost of $\$ 122.00$ for domestic, $\$ 151.94$ for Canadian, and $\$ 142.00$ for international subscribers for Vol. 115 (January-June) and Vol. 116 (July-December). Shipping charges are included. Each bound volume contains a subject and author index and all advertising is removed. Copies are shipped within 60 days after publication of the last issue of the volume. The binding is durable buckram with the Journal name, volume number, and year stamped in gold on the spine. Payment must accompany all orders. Contact Mosby, Inc., Subscription Services, 11830 Westline Industrial Drive, St. Louis, Missouri 63146-3318, USA; phone $800-453-4351$ or 314-453-4351.

Subscriptions must be in force to qualify. Bound volumes are not available in place of a regular Journal subscription. 